\title{
Dietary energy intake is associated with type 2 diabetes risk markers in children
}

\author{
A. S. Donin ${ }^{1}$, C. M. Nightingale ${ }^{1}$, C. G. Owen ${ }^{1}$, A. R. Rudnicka ${ }^{1}$, S. A. Jebb ${ }^{2}$, G. L. Ambrosini ${ }^{2}$, \\ A. M. Stephen ${ }^{2}$, D. G. Cook ${ }^{1}$ and P. H. Whincup ${ }^{1}$ \\ ${ }^{1}$ Division of Population Health Sciences and Education, St George's, University of London, London, UK and ${ }^{2}$ Medical \\ Research Council Human Nutrition Research, Cambridge, UK
}

Type 2 diabetes and its precursor obesity are major global public health problems, requiring concerted preventive efforts ${ }^{(1)}$. Although energy intake, energy density and nutrient intakes are implicated in type 2 diabetes risk in adults, little is known about their influence on emerging obesity and type 2 diabetes risk in childhood. We therefore examined these associations in a multi-ethnic population of children. This is a cross-sectional study of 2017 children predominantly of white European, South Asian and black African-Caribbean origin aged 9-10 years. Children provided detailed 24 hour dietary recalls, measurements of body composition and a fasting blood sample for measurements of serum glucose, HbA1c and fasting insulin; HOMA insulin resistance was also derived.

After the removal of under and over reporters of energy intake, energy intake was positively associated with insulin resistance, HbA1c, glucose and fat mass index; energy density was positively associated with insulin resistance and fat mass index. Individual nutrients showed no associations with any type 2 diabetes risk markers. In mutually adjusted analyses, the associations for energy intake remained while those for energy density became non-significant. Additional adjustment for fat mass index reduced the associations for insulin resistance and $\mathrm{HbA} 1 \mathrm{c}$ by about $40 \%$; the association for fasting glucose was only slightly reduced. Adjustment for differences in total energy intake reduced the South Asian - white European differences in insulin resistance, HbA1c and fasting glucose and the black African-Caribbean-white European differences in insulin resistance and $\mathrm{HbA1c}$.

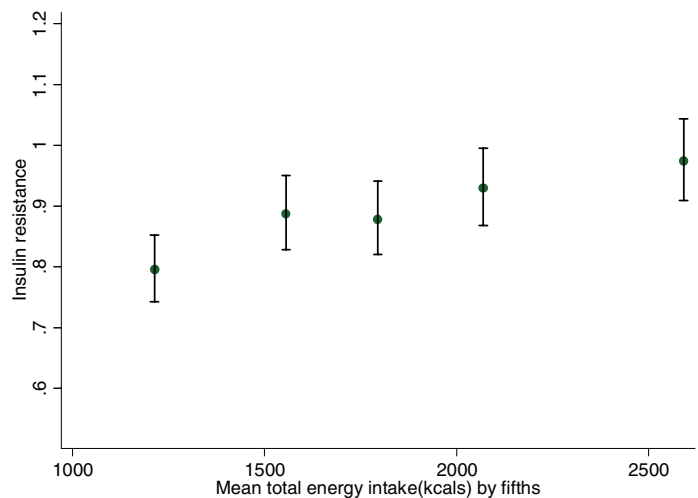

Figure 1a: HOMA insulin resistance by fifths of mean resistance by fifths of total energy in all participants (2017 children).

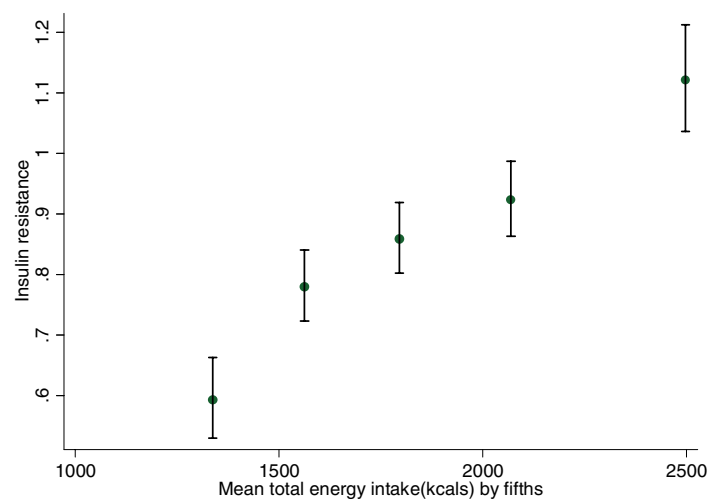

Figure 1b: HOMA insulin mean total energy intakes exclude miss-reporters.

Higher total energy intake was strongly associated with high levels of insulin resistance and may help to explain emerging ethnic differences in type 2 diabetes risk in childhood. These results are consistent with findings from bariatric surgery patients ${ }^{(2)}$ and with other population-based observations on the effects of changes in energy intake on diabetes risk ${ }^{(3)}$. Further study is needed to establish whether reducing energy intake produces favourable changes in insulin resistance and circulating glucose levels.

This work was supported by Diabetes UK research grant (grant reference BDA 11/0004317).

1. Zhang P, Zhang X, Brown J, et al. (2010) Diabetes Res Clin Pract 87, 293-301.

2. Gumbs AA, Modlin IM, Ballantyne GH (2005) Obes Surg 15, 462-473.

3. Franco M, Ordunez P, Caballero B, et al. (2007) Am J Epidemiol 166, 1374-1380. 